\title{
Evolution and Composition of Reusable Assets in Product-Line Architectures: A Case Study
}

\author{
Jan Bosch \\ University of Karlskrona/Ronneby \\ Department of Computer Science and Business Administration \\ S-372 25 Ronneby, Sweden \\ e-mail:Jan.Bosch@ide.hk-r.se \\ www:http://www.ide.hk-r.se/ bosch
}

Key words: Reusable assets, product-line architectures, software composition, software evolution, case study

\begin{abstract}
In this paper, a case study investigating the experiences from evolution and modification of reusable assets in product-line architectures is presented involving two Swedish companies, Axis Communications $\mathrm{AB}$ and Securitas Larm AB. Key persons in these organisations have been interviewed and information has been collected from documents and other sources. The study identified problems related to multiple versions of reusable assets, dependencies between assets and the use of assets in new contexts. The problem causes have been identified and analysed, including the early intertwining of functionality, the organizational model, the time to market pressure, the lack of economic models and the lack of encapsulation boundaries and required interfaces.
\end{abstract}

\section{INTRODUCTION}

Product-line architectures have received attention in research, but even more so in industry. Many companies have moved away from developing software from scratch for each product and instead focused on the commonalities between the different products, and capturing those in a product-line architecture and an associated set of reusable assets. This is, especially in the Swedish industry, a logical development since software is

The original version of this chapter was revised: The copyright line was incorrect. This has been corrected. The Erratum to this chapter is available at DOI: 10.1007/978-0-387-35563-4_35 
an increasingly large part of products and often defines the competitive advantage. When moving from a marginal to a major part of products, the required effort for software development also becomes a major issue and industry searches for ways to increase reuse of existing software to minimize product-specific development and to increase the quality of software.

A number of authors have reported on industrial experiences with product-line architectures. In [SEI 97], results from a workshop on product line architectures are presented. Also, [Macala et al. 96] and [Dikel et al. 97] describe experiences from using product-line architectures in an industrial context. The aforementioned work reports primarily from large, American software companies, often defense-related, which are not necessarily representative of the software industry as a whole, especially European small- and medium-sized enterprises.

We have performed a case study of product-line architectures involving two Swedish software development organisations: Axis Communications $\mathrm{AB}$ and Securitas Larm AB. The former develops and sells network-based products, such as printer, scanner, camera, and storage servers, whereas the latter company produces security- and safety-related products such as firealarm, intruder-alarm, and passage control systems. Since the beginning of the ' $90 \mathrm{~s}$, both organisations have moved towards product-line architecture based software development, especially through the use of object-oriented frameworks as reusable assets. In an earlier paper [Bosch 98c], we reported on the technological, process, organizational and business problems and issues related to product-line architectures. In this paper, we focus on the use, evolution, composition and reuse of assets that are part of a product-line architecture. Since the involved organisations have considerable experience using this approach, we report on their way of organising software development, the obtained experiences and the identified problems.

The contribution of this paper is, we believe, its provision of exemplars of industrial organisations in software industry that can be used for comparison or as inspiration. In addition, the experiences and problems surrounding reusable assets provide, at least partly, a research agenda for the software architecture and software reuse communities.

The remainder of the paper is organised as follows. In the next section, the research method used for the case study is briefly described. The two companies forming the focus of the case study are described in section 3 . Section 4 discusses the differences in perception of product-line architectures and reusable assets in academia and industry. The problems identified during data collection are discussed in section 5 and their causes are analysed in section 6 . Section 7 discusses related work and the paper concludes in section 8 . 


\section{CASE STUDY METHOD}

The goal of the study was twofold: first, our intention was to get an understanding of the problems and issues surrounding reusable assets part that are part of a product-line architecture in "normal" software development organisations, i.e., organisations of small to average size, i.e., tens or a few hundred employees, and unrelated to the defense industry. Second, our goal was to identify those research issues that are most relevant to software industry with respect to reusable assets in product-line software architectures.

The most appropriate method to achieve these goals, we concluded, was interviews with the system architects and technical managers at software development organisations. Since this study marks the start of a three year government-sponsored research project on software architectures involving our university and three industrial organisations, i.e., Axis Communications, Securitas Larm, and Ericsson Mobile Communications, the interviewed parties were taken from this project. The third organisation, a business unit within Ericsson Mobile Communications, is a recent start-up and has not yet produced product-line architectures or products. A second reason for selecting these companies was that we believe them to be representative of a larger category of software development organisations. These organisations develop software that is to be embedded in products also involving hardware and mechanics, are of average size (e.g., development departments of 10 to 60 engineers), and develop products sold to industry or consumers.

The interviews were open and rather unstructured, although a questionnaire was used to guide the process. The interviews were videotaped for further analysis afterwards and in some cases documentation from the company was used to complement the interviews. The interviews often started with a group discussion and were later complemented with interviews with individuals for deeper discussions on particular topics.

\section{CASE STUDY ORGANISATIONS}

\subsection{Case 1: Axis Communications AB}

Axis Communications started its business in 1984 with the development of a printer server product that allowed IBM mainframes to print on nonIBM printers. Up to then, IBM maintained a monopoly on printers for their computers, with consequent price settings. The first product was a major success that established the base of the company. In 1987, the company developed the first version of its proprietary RISC CPU that provided better 
performance and cost-efficiency than standard processors for their datacommunication oriented products. Today, the company develops and introduces new products on a regular basis. At the beginning of the ' 90 s, object-oriented frameworks were introduced into the company and since then a base of reusable assets is maintained from which most products are developed.

Axis develops IBM-specific and general printer servers, CD-ROM and storage servers, network cameras, and scanner servers. The latter three products, in particular, are built using a common product-line architecture and reusable assets. In figure 1, an overview of the product-line and product architectures is shown. The organisation is more complicated than the standard case with one product-line architecture (PLA) and several products below this product-line. In the Axis case, there is a hierarchical organisation of PLAs, with the product-line architecture at the top and the product-group architectures (e.g., the storage-server architecture) at the next lower level. The focus of the case study is on the marked area in the figure, although the other parts are discussed briefly as well. The primary reusable assets for Axis include object-oriented frameworks for file systems and network protocols, but several smaller frameworks are used as well.

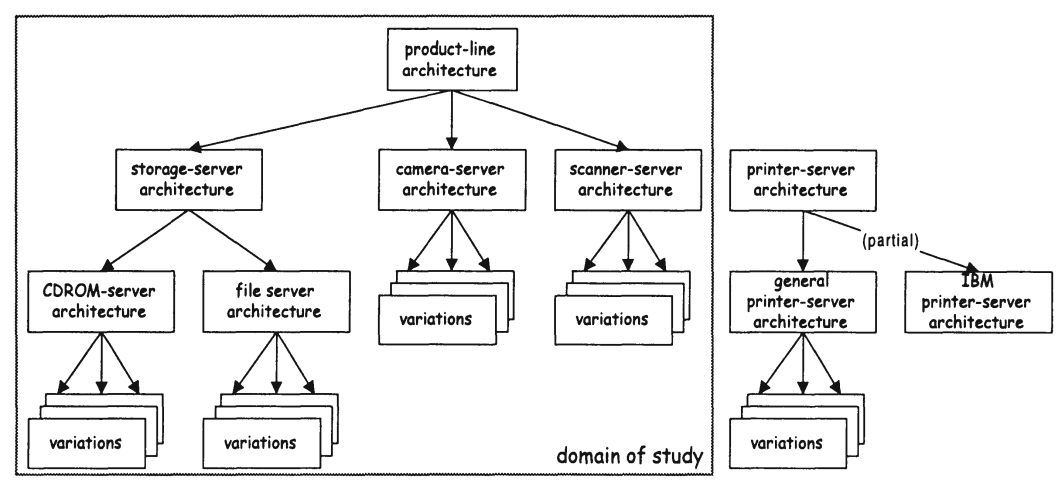

Figure 1. Product-line and product software architectures in Axis Communications

\subsection{Case 2: Securitas Larm AB}

Securitas Larm AB (formerly TeleLarm AB) develops, sells, installs and maintains safety and security systems such as fire-alarm systems, intruder alarm systems, passage-control systems, and video surveillance systems. The company's focus is especially on larger buildings and complexes, requiring integration between the aforementioned systems. Therefore, Securitas has a fifth product unit developing integrated solutions for customers including all 
or a subset of these systems. In figure 2 , an overview of the products is presented.

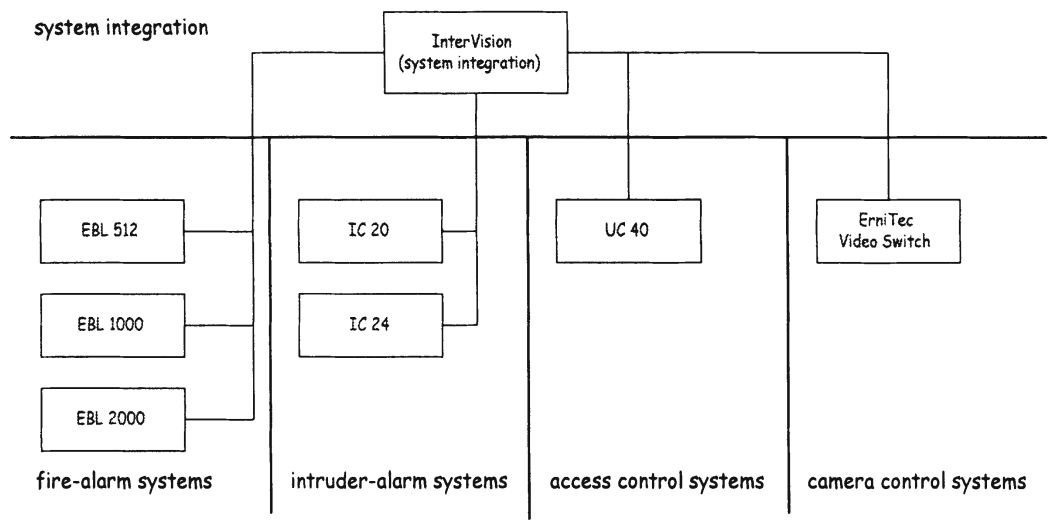

Figure 2. Securitas Larm Product Overview

Securitas uses a product-line architecture only for their fire-alarm products, in practice only the EBL 512 product, and traditional approaches in the other products. However, due to the success in the fire-alarm domain, the intention is to expand the PLA in the near future to include the intruderalarm and passage-control products as well.

\section{PRODUCT-LINE ARCHITECTURES AND REUSABLE ASSETS}

An important issue we identified during this case study and our other cooperative projects with industry is that there exists a considerable difference between the academic perception of software architecture and reusable assets and the industrial practice. It is important to explicitly discuss these differences because the problems described in the next section are based on the industrial rather than the academic perspective. It is interesting to note that sometimes the problems that are identified as the most important and difficult by industry are not identified (or viewed as non-problems) by academia.

Table 1 lists the academic and industrial interpretations of the notion of product-line architecture. The main differences are related to the definition of architectures, the use of first-class connectors, and the use of specialised languages. 
Table 1. Academic versus industrial view of software architecture

\begin{tabular}{|l|l|}
\hline \multicolumn{1}{|c|}{ Research } & \multicolumn{1}{c|}{ Industry } \\
\hline Architecture is explicitly defined. & $\begin{array}{l}\text { Mostly conceptual understanding of } \\
\text { architecture. Minimal explicit definition, often } \\
\text { through notations. }\end{array}$ \\
\hline $\begin{array}{l}\text { Architecture consists of components } \\
\text { and first-class connectors. }\end{array}$ & $\begin{array}{l}\text { No explicit first-class connectors (sometimes } \\
\text { ad-hoc solutions for run-time binding and glue } \\
\text { code for adaptation between assets). }\end{array}$ \\
\hline $\begin{array}{l}\text { Architectural description languages } \\
\text { (ADLs) explicitly describe } \\
\text { architectures and are used to } \\
\text { automatically generate applications. }\end{array}$ & $\begin{array}{l}\text { Programming languages (e.g., C+++) and script } \\
\text { languages (e.g., Make) used to describe the } \\
\text { configuration of the complete system. }\end{array}$ \\
\hline
\end{tabular}

For reusable assets, one can identify a similar difference between the academic and industrial understanding of the concepts. In table 2, an overview is presented comparing the two views. The main differences are related to, among others, the assumed black-box nature, the component interface, and variability.

Table 2. Academic versus industrial view of reusable assets

\begin{tabular}{|l|l|}
\hline \multicolumn{1}{|c|}{ Research } & \multicolumn{1}{c|}{ Industry } \\
\hline $\begin{array}{l}\text { Reusable assets are black-box } \\
\text { components. }\end{array}$ & $\begin{array}{l}\text { Assets are large pieces of software (sometimes more } \\
\text { than 80 KLOC) with a complex internal structure and } \\
\text { no enforced encapsulation boundary, e.g., object- } \\
\text { oriented frameworks. }\end{array}$ \\
\hline $\begin{array}{l}\text { Assets have narrow interface } \\
\text { through a single point of access. }\end{array}$ & $\begin{array}{l}\text { The asset interface is provided through entities, e.g., } \\
\text { classes in the asset. These interface entities have no } \\
\text { explicit differences to non-interface entities. }\end{array}$ \\
\hline $\begin{array}{l}\text { Assets have few and explicitly } \\
\text { defined variation points that are } \\
\text { configured during instantiation. }\end{array}$ & $\begin{array}{l}\text { Variation is implemented through configuration and } \\
\text { specialisation or replacement of entities in the asset. } \\
\text { Sometimes multiple implementations (versions) of } \\
\text { assets exist to cover variation requirements }\end{array}$ \\
\hline $\begin{array}{l}\text { Assets implement standardized } \\
\text { interfaces and can be traded on } \\
\text { component markets. }\end{array}$ & $\begin{array}{l}\text { Assets are primarily developed internally. Externally } \\
\text { developed assets go through considerable (source } \\
\text { code) adaptation to match the product-line } \\
\text { architecture requirements. }\end{array}$ \\
\hline $\begin{array}{l}\text { Focus is on asset functionality } \\
\text { and on the formal verification of } \\
\text { functionality. }\end{array}$ & $\begin{array}{l}\text { Functionality and quality attributes, e.g., } \\
\text { performance, reliability, code size, reusability and } \\
\text { maintainability, have equal importance. }\end{array}$ \\
\hline
\end{tabular}

\section{PROBLEMS}

Based on the interviews and other documentation collected at the organisations part of this case study, we have identified a number of problems related to reusable assets that we believe to have relevance in a 
wider context than just these organisations. In the remainder of this section, the problems that were identified during the data collection phase of the case study are presented. For each problem, a problem description is presented, illustrated by an example from one of the case-study companies. The problems are categorized into three categories, related to multiple versions of assets, dependencies between assets, and the use of assets in new contexts.

\subsection{Multiple versions of assets}

Product-line architectures have associated reusable assets that implement the functionality of architectural components. These assets can be very large and contain up to a hundred KLOC or more. Consequently, they represent considerable investments (multiple man-years in certain cases). Therefore, it was surprising to identify that in some cases, the interviewed companies maintained multiple versions (implementations) of assets in parallel. One can identify at least four situations where multiple versions are introduced.

\subsubsection{Conflicting quality requirements}

The reusable assets are generally optimized for particular quality attributes such as performance or code size. Different products in the product line, even though they require the same functionality, may have conflicting quality requirements. These requirements may have so high a priority that no single component can fulfil them all. The reusability of the affected asset is then restricted to just one or a few of the products while other products require another implementation of the same functionality.

For example, in Axis, the printer server product was left out of the product-line architecture (although it can be considered to be a PLA on its own, with more than 20 major variations) because minimizing the binary code size is the driving quality attribute for the printer server whereas performance and time to market are the driving quality attributes for the other network-server products.

Our impression is that when products in the product-line are at different points in their lifecycle, there is a tendency to have multiple versions of assets. This is because the driving quality attributes of a product tend to change during its lifecycle from feature- and time-to-market driven to costand efficiency-driven (see also [SEI 97]).

\subsubsection{Variability implemented through versions}

Certain types of variability are difficult to implement through configuration or compiler switches since the effect of a variation spreads out 
throughout the reusable asset. An example is different contexts, such as the operating system, for an asset. Although it might be possible to implement all variability through, for example, \#ifdef statements, often it is decided to maintain two different versions.

The above printer server example can also be used here. The different versions of assets actually implement different variability selections.

\subsubsection{High-end versus low-end products}

The reusable asset should contain all functionality required by the products in the product-line, including the high-end products. The problem is that low-end products, generally requiring a restricted subset of the functionality, pay for the unused functionality in terms of code size and complex interfaces. Especially for embedded systems where the hardware costs play an important role in the product price, the software engineers may be forced to create a low-end, scaled-down version of the asset to minimize the overhead for low-end products.

Two versions of the file-system framework have been used in Axis in different products. The scanner and camera products used a scaled down version of the file system framework, only implementing a memory-based pseudo file system, whereas the CD-Rom and Jaz drive products used the full-scale file system, implementing a variety of file-system standards. The scanner and camera product develpoers had no interest in incorporating the complete asset since it required more memory than strictly necessary, leading to increased product cost.

\subsubsection{Business unit needs}

Especially in the organizational model used by Axis, where the business units are responsible for asset evolution, assets are sometimes extended with very product-specific code, or code only tested for one of the products in the product-line. The problems caused by this create a tendency within the affected business units to create their own copy of the asset and maintain it solely for their own product. This minimizes the dependency on the shared product-line architecture and solves the problems in the short term, but in the long term it generally does not pay off. We have seen several instances of cases where business units had to rework considerable parts of their code to incorporate a new version of the evolved shared asset that contained functionality that needed to be incorporated in their product also.

The aforementioned file system framework example is also an example of a situation where business-unit needs resulted in two versions of an asset. At a later stage, the full-scale file system framework had evolved and the 
scanner and camera products wanted to incorporate the additional functionality. In order to achieve that, the product-specific code of both products had to be reworked in order to incorporate the evolved file system framework.

\subsection{Dependencies between assets}

Since the reusable assets are all part of a product-line architecture, they tend to have dependencies between them. Although dependencies between assets are necessary, assets often have dependencies that could have been avoided by another modularization of the system or a more careful asset design. From the examples at the studied companies, we learned that the initial design of assets generally defines a small set of required and explicitly defined dependencies. It is often during evolution of assets that unwanted dependencies are created. Addition of new functionality may require extension of more than one asset; in the process dependencies are often created between the assets that implement the functionality. These new dependencies could often have been avoided by another decomposition of the architecture. They have a tendency to be implicit, in that their documentation is often minimal, and the software engineer encounters the dependency late in the development process. Dependencies in general, but especially implicit dependencies, reduce the reusability of assets in different contexts, but also complicate the evolution of assets within the PLA since each extension of one asset may affect multiple other assets. Based on our research at Axis and Securitas, we have identified three situations where new, often implicit, dependencies are introduced:

\subsubsection{Component decomposition}

With the development of the product-line architecture, generally the sizes of the reusable assets also increase. Companies often have some optimal size for an asset component, so that it can be maintained by a small team of engineers (e.g., it captures a logical piece of domain functionality, etc.). With the increasing size of asset components, there is a point where a component needs to be split into two components. These two components, initially, have numerous relations to each other, but even after some redesign several dependencies often remain because the initial design did not modularize the behaviour of by the two components. One could, obviously, redesign the functionality of the components completely to minimize the dependencies, but the required effort is generally not feasible in development organizations. 
To give an example from Axis: at some point, it was decided that the file system asset should be extended with functionality for authorisation. To implement this, it proved to be necessary to also extend the protocol asset with some functionality. This created yet another dependency between the file system and the protocol assets, making it harder to reuse them separately. Currently, the access functionality has been broken out of the file system and protocol assets, and defined as a separate asset, but some dependencies between the three assets remain.

\subsubsection{Extensions cover multiple assets}

Extension of the product-line architecture stems from new functional requirements that need to be incorporated in the existing functionality. Often, the required extension to the product line covers more than one asset. During implementation of the extension, it is very natural to add dependencies between the affected assets since one is working on functionality that is perceived as one piece, even though it is divided over multiple assets.

The authorisation access extension to the Axis PLA provides, again, an excellent example. At first, the access functionality was added to the file system and protocol assets. However, the protocol framework contained the protocol user classes that were needed by the access functionality in the file system framework, leading to strong dependencies between the two frameworks. At a later stage, the authorisation access was separated from the two assets and represented as a single asset, thereby decreasing the dependencies.

\subsubsection{Asset extension adds dependency}

As mentioned, the initial design of a PLA generally minimizes dependencies between its components. Evolution of an asset component may cause this component to require information from an earlier unrelated component. If this dependency had been known during the initial PLA design, then the functionality would have been modularized differently and the dependency would have been avoided.

In the protocol framework in the Axis PLA, most of the implemented protocols use a layered organisation in which process packets that are sent up and down the protocol layers. These communication packets are nested in the sense that each lower-level protocol layer declares a new packet and adds the received packet as an argument. At some point, the implementation of new functionality required methods of the most encapsulated packet object to refer to data in one of the packets higher up in the encapsulation 
hierarchy, introducing a very unfortunate dependency between the two packets.

\subsection{Assets in new contexts}

Since assets represent considerable investments, the goal is to use assets in as many products and domains as possible. However, a new context differs in one or more aspects from the old context, causing a need for the asset to be changed in order to fit. Two main issues in the use of assets in new contexts can be identified.

\subsubsection{Mixed behaviour}

An asset is developed for a particular domain, product category, operating context, and set of driving quality requirements. Consequently, it often proves to be hard to apply the asset in different domains, products, or operating contexts. The design of assets often hard-wires design decisions concerning these aspects unless the type of variability is known and required at design time.

The main asset for Securitas is the highly successful fire-alarm system. In the near future, Securitas intends to develop a similar asset for the domain of intruder-alarm systems. Since the domains have many aspects in common, their intention is to reuse the fire-alarm asset and apply it to the intruder alarm domain, rather than developing the asset from scratch. However, initial investigations show that the domain change for the asset is not a trivial endeavour.

\subsubsection{Design for required variability}

It is recommended best practice that reusable assets be designed to support only the variability requested in the initial requirement specification, e.g., [Jacobson et al. 97]. However, a new context for a reusable asset often also requires new variability dimensions. One cannot expect that assets are designed to include all foreseeable forms of variability, but they should be designed so that the introduction of new variability requires minimal effort.

The application of the fire-alarm framework in the intruder-alarm domain serves as an example here. These systems share, to a large extent, the same operating context and quality requirements. However, since the fire-alarm domain functionality is hard-wired in the framework design, and the intruder alarm domain has different requirements and concepts, one is forced to introduce variability for application-domain functionality. 


\section{CAUSE ANALYSIS}

The problems discussed in the previous section represent an overview of the issues surrounding the use of reusable assets in a product-line architecture. We have analysed these problems in their industrial context and have identified what we believe to be the primary underlying causes of these problems. In the remainder of this section, these causes are discussed.

\subsection{Early intertwining of functionality}

The functionality of a reusable asset can be categorized into functionality related to the application domain, the quality attributes, the operating context, and the product-category. Although these different types of functionality are treated separately at design time, both in the design model and the implementation they tend to be mixed. Hence it is generally hard to change one of the functionality categories without extensive reworking of the asset. Both the state-of-practice as well as leading authors on reusable software (e.g., [Jacobson et al. 97]), design for required variability only. That is, only the variability known at asset-design time is incorporated in the asset. Since the requirements evolve constantly, requirement changes related to the domain, product category, or context generally appear after design time. Consequently, it often proves hard to apply the asset in the new environment.

The early intertwining of functionality is a primary cause of several of the problems discussed in the previous section. Multiple versions of assets are required because the different categories of functionality cannot be separated in the implementation and implemented through variability. Also, the use of an asset in a new context is complicated by the mixing of functionality.

Companies try to avoid mixed functionality primarily through design. For instance, the use of layers, even in asset design, to separate operatingcontext-dependent from context-independent functionality, avoids the mixing. Also, several design patterns [Gamma et al. 94, Buschmann et al. 96] support separation of different types of functionality and support the introduction of variability.

Research issues. The primary research issue is to find approaches that allow for late composition of different types of functionality. Examples of this can be found in the Draco system [Neighbors 89], [Batory \& O'Malley 92] approach to hierarchical software systems, parameterized programming [Goguen 96], aspect-oriented programming [Kiczales et al. 97] and in the layered object model [Bosch 98a] and [Bosch 98b]. In addition, design 
solutions, such as design patterns, that successfully separate functionality should be a continuing topic of research.

\subsection{Organization}

Both Securitas and Axis have explicitly decided against the use of separate domain engineering units. The advantages of separate domain engineering units, such as being able to spend considerable time and effort on thorough designs of assets, were generally recognised. On the other hand, people felt that a domain engineering group could easily get lost in wonderfully high abstractions and highly reusable code that did not quite fulfil the requirements of the application engineers. In addition, having explicit groups for domain and application engineering requires a relatively large software development department consisting of at least fifty to a hundred engineers.

Nevertheless, several of the problems discussed earlier can be related to the lack of independent domain engineering. Business units focus on their own quality attributes and design for achieving those during asset extension. Because of that, multiple versions of assets may be created where a domain engineering unit might have found solutions allowing for a single version. In addition, asset extension without sufficient focus on the product-line as a whole may introduce more dependencies than strictly necessary, complicating the use of assets as well as their reuse in new contexts.

Solutions exist to minimize the negative effects of organizational structures. At Axis, so-called asset redesigns are performed when a consensus is present that an asset needs to be reorganised. During an asset redesign, the software architects from the business units using the asset gather to redesign the asset in order to improve its structure. As a complement, both Axis and Securitas have responsibility for each asset, and evolution of assets has to be approved by them. However, because of timeto-market pressures, there is sometimes a need to accept less-than-optimal solutions. Thirdly, to improve on these issues, management must be willing to occasionally relieve some time-to-market pressure, accepting delay of one product so that subsequent products can enter the market sooner.

Research issues. The primary research issue concerns the processes surrounding asset evolution. More case studies and experimentation are required to gather evidence of working and failing processes, and mandatory and optional steps. In addition, one can conclude that it is unclear when an organisation should have separate domain engineering units rather than performing asset development in the application engineering units. Research is required for the collection of evidence on optimal organizational structures 
and identification and evaluation of approaches to minimize the negative effects of organizational choices.

\subsection{Time to market}

A third important cause for the problems related to reusable assets at the interviewed companies is the time-to-market (TTM) pressure. Getting out new products and subsequent versions of existing products is very high up on the agenda, thereby sacrificing other topics. The problem most companies are dealing with is that products appearing late on the market will lead to diminished market share or, in the worst case, to no market penetration at all. However, this all-or-nothing mentality leads to an extreme focus on shortterm goals, while ignoring long term goals. Sacrificing some time-to-market for one product may lead to considerable improvements for subsequent products, but this is generally not appreciated.

The TTM pressure causes several of the problems discussed earlier. This is primarily because software engineers do not have the time to reorganise the assets to minimize dependencies or to generalize functionality. Asset evolution is often implemented as quick fixes, thereby decreasing the usability of the asset in future contexts.

To address the problems resulting from TTM pressure, it is important for software development organizations to regard the development of a productline architecture and associated assets as a strategic issue, with decisions being made at the appropriate level. The consequences for the time-tomarket of products under development should be balanced against the future returns. Finally, taking a time-out for asset redesign is necessary periodically to "clean up."

Research issues. Decisions related to TTM for products are made based on a business case and these, rather relevant, research issues are outside the software engineering domain. However, two issues can be identified: the lack of economic models (described in the next section) and design techniques that minimize the effort required for extending assets without diminishing their future applicability.

\subsection{Economic models}

As mentioned earlier in the paper, reusable assets may represent investments of up to several man-years of implementation effort. For most companies, such assets represent a considerable amount of capital, but both engineers and management are not always aware of that. For instance, an increasing number of dependencies (especially implicit dependencies) 
between assets is a sign of accelerated aging of software and, in effect, decreases the value of the assets. However, since no economic models are available that visualise the effects of quick fixes causing increased dependencies, it is hard to establish the economic losses of these dependencies. In addition, reorganisation of software assets that have been degrading for some while is often not performed because no economic models are available to visualize the return on investment.

The lack of economic models influences several of the identified problems. In general, one can recognize a lack of forces against time-tomarket pressure because no business case for sound engineering (versus deadline-driven hacking of software) can presented.

Research issues. One can identify a need for economic models in two situations. Firstly, models are needed for calculating the economic value of an asset, based on the investment (man hours) but also on the value of the asset for future product development and/or for an external market. Secondly, models are needed for visualising the effects of various types of changes and extensions to the asset value. These models could be used to visualise the effects of quick fixes and implicit dependencies on the asset value.

\subsection{Encapsulation boundaries and required interfaces}

Although many of the issues surrounding product-line architectures are non-technical in nature, there are technical issues as well. The lack of encapsulation boundaries that encapsulate reusable assets and enforce explicitly defined points of access through a narrow interface is a cause of a number of the identified problems. In section 4 we discussed the difference between the academic and the industrial view of reusable assets. Some of the assets at the interviewed companies are large object-oriented frameworks with a complex internal structure. The traditional approach is to distinguish between interface classes and internal classes. The problem is that this approach lacks support from the programming language, requiring software engineers to adhere to conventions and policies. In practice, especially under strong time-to-market pressure, software engineers will go beyond the defined interface of assets, creating dependencies between assets that may easily break when the internal implementation of assets is changed. In addition, these dependencies tend to be undocumented or only minimally documented.

A related problem is the lack of required interfaces. Interface models generally describe the interface provided by a component, but not the interfaces it requires from other components for its correct operation. Since 
dependencies between components can be viewed as instances of bindings between required and provided interfaces, one can conclude that it is hard to visualize dependencies if the necessary elements are missing.

The lack of encapsulation boundaries and required interfaces primarily causes problems related to component dependencies. For instance, component decomposition is complicated since the new part-components can continue to refer to each other without explicit visibility.

As mentioned, companies address these issues by establishing conventions and policies, but these tend to be broken in practice. Documentation of the assets and inspection of design documents, the implementation and the documentation of assets helps enforce the conventions and policies.

Research issues. The primary research issue to address this cause is to find approaches to encapsulation boundaries that are more open than the blackbox component models, but provide protection for the private entities that are part of the assets. Also, more research on the specification and semantics of required interfaces is needed. One example of an existing model is described in [Batory \& O'Malley 92]. A second example is the layered object model where an "acquaintance-based" approach is presented that allows for specifying required interfaces and binding these interfaces to other components [Bosch 98b].

\section{RELATED WORK}

Tools, techniques, and approaches to the development of families of software products have been proposed by a number of authors. LIL [Goguen 86] is an example of a module interconnection language (MIL) that describes component (or module) based systems. In [Neighbors 89], the Draco approach is discussed that, although not using the same terminology, identifies the basic structures of software development based on reuse of domain designs and implementations. [Perry 89] discussed the Inscape environment, focusing on software evolution and problems of scale (i.e., complexity, programming-in-the-large, and programming-in-the-many). [Goguen 96] discusses parameterized programming to instantiate generic descriptions with domain-specific components. [Batory \& O'Malley 92, Batory \& Geraci 97] discuss a hierarchical component-based model that facilitates the development of families of systems. [Biggerstaff 94] discusses a basic problem in component-based software development, i.e., scaling, and identifies some of the problems discussed in this paper. 
With respect to product-line architectures, a number of authors have studied their industrial use. [Macala et al. 96] discuss a demonstration project using product-line development in Boeing in cooperation with the US Navy as part of the STARS initiative. The authors identify four elements of product-line development, i.e., process-driven, domain-specific, technology support, and architecture-centric. The lessons learned during the projeci are discussed and a set of recommendations is presented. [Dikel et al. 97] discuss lessons learned from using a product-line architecture in Nortel and present six principles: focusing on simplification, adapting to future needs, establishing architectural rhythm, partnering with stakeholders, maintaining vision, and managing risks and opportunities. The report from the product-line practice workshop held by the SEI [SEI 97] presents an overview of the state-of-practice in a number of large software development organisations. Contextual, technology, organizational and business aspects are discussed and a number of critical factors are identified, including deep domain expertise, well-defined architecture, distinct architect, solid business case, management commitment and support and domain engineering unit.

An interesting difference between the papers mentioned above and the results of our study is the perceived necessity of separate domain engineering units. The organisations of our case study explicitly decided against separate domain engineering units. Also [Simos 97] reacts against using domain engineering units and suggests a unified lifecycle model.

[Jacobson et al. 97] presents a complete approach to institutionalizing software reuse in an organisational context, including technology, process, and business aspects. The book is based primarily on experiences from the HP and Ericsson context and contains excellent suggestions also applicable to the interviewed companies.

The Taligent frameworks [Taligent 95] provide various interfaces to each framework, including a client API and a customization API. However, no approaches to language support for high-level encapsulation boundaries are presented. [Szyperski 97] presents an overview of component-oriented programming and discusses the necessity of "required interfaces" in addition to the "provided interfaces". He recognises the necessity of required interfaces, but concludes that current commercial component models focus on provided interfaces only.

\section{CONCLUSIONS}

The notion of product-line architectures has received attention especially in industry since it provides a means to exploit the commonalities between related products and thereby reduce development cost and increase quality. 
In this paper, we have presented a case study involving two Swedish companies, Axis Communications $\mathrm{AB}$ and Securitas Larm $\mathrm{AB}$, that use product-line architectures in their product development. Key persons in these organisations have been interviewed and information has been collected from documents and other sources. The goal of the case study was to examine the use, evolution, composition and reuse of assets in a product-line architecture.

In the previous sections, a number of problems and underlying causes are described that were identified in the case study organisations and generalised to a wider context. We have identified three categories of problems related to reusable assets:

1. the existence of multiple versions of assets

2. dependencies between assets

3. the use of assets in new contexts

In the analysis we focus on the causes that we believe underlie the identified problems. The identified causes include

- the early intertwining of functionality

- the organizational structure

- the time-to-market pressure

- the lack of economic models

- the lack of explicit encapsulation boundaries and required interfaces.

In conclusion, product-line architectures can be, and are being, successfully applied in small- and medium-sized enterprises. The studied organisations are struggling with a number of difficult problems and challenging issues, but the general consensus is that a product-line architecture approach is beneficial, if not crucial, for the continued success of these organisations.

\section{ACKNOWLEDGEMENTS}

The author would like to thank the software architects and engineers and technical managers at Axis Communications $\mathrm{AB}$ and Securitas Larm AB, in particular Torbjörn Söderberg and Rutger Pålsson. Thanks also to the anonymous reviewers for their comments.

\section{REFERENCES}

[Batory \& Geraci 97] D. Batory and B.J. Geraci, 'Validating Component Compositions and Subjectivity in GenVoca Generators', IEEE Transactions on Software Engineering, February 1997, 67-82. 
[Batory \& O'Malley 92] D. Batory and S. O'Malley, 'The Design and Implementation of Hierarchical Software Systems with Reusable Components', ACM Transactions on Software Engineering and Methodology, October 1992.

[Biggerstaff 94] T. Biggerstaff, 'The Library Scaling Problem and the Limits of Concrete Component Reuse', Third International Conference on Software Reuse, Rio de Janeiro, November 1-4, 1994, 102-110.

[Bosch 98a] J. Bosch, 'Design Patterns as Language Constructs,' Journal of Object-Oriented Programming, Vol. 11, No. 2, pp. 18-32, May 1998.

[Bosch 98b] J. Bosch, 'Object Acquaintance Selection and Binding,' accepted for publication in Theory and Practice of Object Systems, February 1998.

[Bosch 98c] J. Bosch, 'Product-Line Architectures in Industry: A Case Study,' submitted, June 1998.

[Buschmann et al. 96] F. Buschmann, C. Jäkel, R. Meunier, H. Rohnert, M.Stahl, PatternOriented Software Architecture - A System of Patterns, John Wiley \& Sons, 1996.

[Dikel et al. 97] D. Dikel, D. Kane, S. Ornburn, W. Loftus, J. Wilson, 'Applying Software Product-Line Architecture,' IEEE Computer, pp. 49-55, August 1997.

[Gamma et al. 94] E. Gamma, R. Helm, R. Johnson, J.O. Vlissides, Design Patterns Elements of Reusable Object-Oriented Software, Addison-Wesley, 1994.

[Goguen 86] J. Goguen, 'Reusing and Interconnecting Software Components', IEEE Computer, February 1986.

[Goguen 96] J. Goguen, 'Parameterized Programming and Software Architecture', 4th International Conference on Software Reuse, Orlando, Florida, April 1996.

[Jacobson et al. 97] I. Jacobson, M. Griss, P. Jönsson, Software Reuse - Architecture, Process and Organization for Business Success, Addison-Wesley, 1997.

[Johnson \& Foote 88] R. Johnson, B. Foote, 'Designing Reusable Classes,' Journal of ObjectOriented Programming, Vol. 1 (2), pp. 22-25, 1988.

[Kiczales et al. 97] G. Kiczales, J. Lamping, A. Mendhekar, C. Maeda, C. Lopes, J-M. Loingtier, J. Irwin, 'Aspect-Oriented Programming,' Proceedings of ECOOP'97 (invited paper), pp. 220-242, LNCS 1241, 1997.

[Kruchten 95] P.B. Kruchten, 'The 4+1 View Model of Architecture,' IEEE Software, pp. 4250, November 1995.

[Macala et al. 96] R.R. Macala, L.D. Stuckey, D.C. Gross, 'Managing Domain-Specific Product-Line Development,' IEEE Software, pp. 57-67, 1996.

[Neighbors 89] J. Neighbors, 'Draco: A Method for Engineering Reusable Software Components', in T.J. Biggerstaff and A. Perlis, eds., Software Reusability, AddisonWesley/ACM Press, 1989.

[Perry 89] D. Perry, 'The Inscape Environment', Proceedings ICSE 1989, 2-12.

[SEI 97] L. Bass, P. Clements, S. Cohen, L. Northrop, J. Withey, 'Product Line Practice Workshop Report,' Technical Report CMU/SEI-97-TR-003, Software Engineering Institute, June 1997.

[Simos 97] M.A. Simos, 'Lateral Domains: Beyond Product-Line Thinking,' Proceedings Workshop on Institutionalizing Software Reuse (WISR-8), 1997.

[Szyperski 97] C. Szyperski, Component Software - Beyond Object-Oriented Programming, Addison-Wesley, 1997.

[Taligent 95] Taligent, The Power of Frameworks, Addison-Wesley, 1995. 